Research Paper

\title{
Fabrication of cellulose acetate/polybenzoxazine cross-linked electrospun nanofibrous membrane for water treatment
}

\author{
Yelda Ertas, Tamer Uyar* \\ Institute of Materials Science \& Nanotechnology, UNAM-National Nanotechnology Research Center Bilkent University, Ankara, 06800, Turkey
}

\section{A R T I C L E I N F O}

\section{Keywords:}

Cellulose acetate

Electrospinning

Nanofibers

$\mathrm{PAH}$ removal

Polybenzoxazine

Water treatment

\begin{abstract}
A B S T R A C T
Herein, polybenzoxazine based cross-linked cellulose acetate nanofibrous membrane exhibiting enhanced thermal/mechanical properties and improved adsorption efficiency was successfully produced via electrospinning and thermal curing. Initially, suitable solution composition was determined by varying the amount of the benzoxazine (BA-a) resin, cellulose acetate (CA) and citric acid (CTR) to obtain uniform nanofibrous membrane via electrospinning. Subsequently, thermal curing was performed by step-wise at $150,175,200$ and $225^{\circ} \mathrm{C}$ to obtain cross-linked composite nanofibrous membranes. SEM images and solubility experiments demonstrated that most favorable result was obtained from the $10 \%(\mathrm{w} / \mathrm{v})$ CA, $5 \%(\mathrm{w} / \mathrm{v})$ BA-a and $1 \%(\mathrm{w} / \mathrm{v})$ CTR composition and cross-linked nanofibrous membrane (CA10/PolyBA-a5/CTR1) was obtained after the thermal curing. Chemical structural changes (ring opening) occurred by thermal curing revealed successful cross-linking of BA-a in the composite nanofibrous membrane. Thermal, mechanical and adsorption performance of pristine CA and CA10/PolyBA-a5/CTR1 nanofibrous membranes were studied. Char yield of the pristine CA nanofibrous membrane has increased notably from 12 to $24.7 \%$ for composite CA10/PolyBA-a5/CTR1 membrane. When compared to pristine CA membrane, CA10/PolyBA-a5/CTR1 nanofibrous membrane has shown superior mechanical properties having tensile strength and Young's modulus of $8.64 \pm 0.63 \mathrm{MPa}$ and $213.87 \pm 30.79 \mathrm{MPa}$, respectively. Finally, adsorption performance of pristine CA and CA10/PolyBA-a5/CTR1 nanofibrous membranes was examined by a model polycyclic aromatic hydrocarbon (PAH) compound (i.e. phenanthrene) in aqueous solution, in which CA10/PolyBA-a5/CTR1 nanofibrous membrane has shown better removal efficiency (98.5\%) and adsorption capacity $(592 \mu \mathrm{g} / \mathrm{g})$.
\end{abstract}

\section{Introduction}

Recent years, many approaches have been proposed to reduce the amount of wastewater produced and improve its quality since it is very essential for human health and environment (Rodriguez, PeraltaHernandez, Goonetilleke, \& Bandala, 2017; Voisin, Bergström, Liu, Mathew, 2017; Ying et al., 2017). Numerous studies have been focused on the development of novel high-performance polymeric membranes for water purification and wastewater treatments. Most polymeric membranes fabricated by the conventional phase inversion method have intrinsic limitations such as low flux, high-fouling tendency and the corresponding pore size distribution for water purification (Bhardwaj \& Kundu, 2010). On the other hand, electrospinning is a simple and versatile nanofiber production technique which can provide fabrication of uniform nanofibrous membranes with nanoscale porous structures (Suja, Reshmi, Sagitha \& Sujith, 2017; Ahmed, Lalia \& Hashaikeh, 2015). Also, polymeric nanofibrous membrane obtained by electrospinning have several characteristics such as high surface area to volume ratio, high density of pores with size range from tens of nanometer to several micrometers, interconnected open pore structure and high permeability (Suja et al., 2017; Ahmed et al., 2015). In addition, the functionalization and improvement of electrospun polymeric nanofibers by simply blending with other types of polymers/ resins in the electrospinning solution to produce composite nanofibrous membranes with enhanced properties could make electrospun nanofiber-based membranes very attractive for filtration.

Typically, membrane materials require some characteristics such as good mechanical property, thermal stability, chemical resistance and ease of processing in order to be used for water filtration and purification. Various polymers, such as polypropylene, polysulfone, poly (ether sulfone), poly(vinylidene fluoride), cellulose acetate, poly(acrylonitrile), and polyamides are good candidates for filtration applications (Miller, Dreyer, Bielawski, Paul \& Freeman, 2016). Among these polymer types, cellulose acetate is one of the widely used polymer type for the production of filtration membrane due to its high hydrophilicity, good toughness, high biocompatibility and resistance to solvents

\footnotetext{
* Corresponding author.

E-mail addresses: uyar@unam.bilkent.edu.tr, tameruyar@gmail.com (T. Uyar).
} 
(Abedini, Mousavi \& Aminzadeh, 2011). As it is known, polymeric nanoporous membranes show superior filtration efficiency with fast permeation and high perm-selectivity, whereas these materials usually exhibit poor tolerance to high temperatures, organic solvents and corrosive environments (Ying et al., 2017). Cellulose acetate or functionalized cellulose acetate nanofibrous membranes posses very good filtration performance to variety of substances such as metal ions (Tian et al., 2011; Ji et al., 2012; Saliba, Gauthier, Gauthier \& Petit-Ramel, 2000; Taha, Wu, Wang \& Li, 2012), dye molecules (Chen, Huang \& Xu, 2011; Wang et al., 2014), polycyclic aromatic hydrocarbons (PAHs) (Celebioglu, Demirci \& Uyar, 2014). However, their thermal and mechanical properties need to be enhanced for the application especially in harsh conditions. Although, several attempts have been done to enhance the filtration efficiency (membrane surface hydrophilicity, water permeability, fouling resistance, etc) of cellulose acetate membranes by blending with other polymers (Sun \& Chen, 2016; Ahmad et al., 2016; Yin et al., 2016; Razzaghi, Safekordi, Tavakolmoghadam, Rekabdar \& Hemmati, 2014; Shenvi, Ismail \& Isloor, 2014; Radha, Shobana, Tarun \& Mohan, 2014; Kumari, Sarkhel \& Choudhury, 2013), nanoparticles (Arthanareeswaran, Devi \& Raajenthiren, 2008; Goh, Ng, Lau \& Ismail, 2015), grapheneoxide (Safarpour, Khataee \& Vatanpour, 2015; Hegab \& Zou, 2015; Chae et al., 2015), carbon nanotubes (Sabir et al., 2015; El Badawi, Ramadan, Esawi \& El-Morsi, 2014; Choi et al., 2015) and epoxy resins (Mahendran, Malaisamy \& Mohan, 2004; Mahendran, Malaisamy \& Mohan, 2002), a few studies were reported to show enhancement of thermal stability or/and mechanical properties of cellulose acetate membranes (Persson, Gekas \& Trägårdh, 1995; Ebert, Fritsch, Koll \& Tjahjawiguna, 2004; Corobea et al., 2016; Huang, Li, Liu \& Zhang, 2015; El-Din, El-Gendi, Ismail, Abed \& Ahmed, 2015; Ahmad et al., 2015; Dasgupta et al., 2014; Abedini et al., 2011). In these studies, inorganic nanomaterials or carbon nanotubes were used as a nanofiller for the enhancement of thermal/mechanical properties.

Polybenzoxazines as a new-class of phenolic type thermoset resin could be good candidate as membrane materials for water filtration. Polybenzoxazines have many intriguing properties such as near-zero volumetric change upon curing, no by-products without any catalysts during curing, low water absorption, high glass transition temperature, high char yield, good mechanical and thermal properties (Ishida \& Agag, 2011). These properties of polybenzoxazines make them good candidate for the production of high performance composite materials not only bulk state but also fabrication at nano-scale. In this study, bisphenol-A and aniline-based benzoxazine monemer (BA-a) was used as a blending resin material with cellulose acetate (CA). Two oxazine rings of BA-a contribute the cross-linking reactions through opening of the oxazine rings by thermal activation and results with highly cross-linked structure with benzene side groups. It is well know that, benzene or cyclic side groups have higher effect on the thermal and mechanical properties compared to aliphatic chains (Ishida \& Agag, 2011). Therefore, BA-a is a very good blending material as a benzoxazine resin to enhance the thermal and mechanical properties of CA nanofibrous membrane.

The polycyclic aromatic hydrocarbons (PAHs) as a large family of fused benzene rings are one of the most widespread pollutants. These compounds have high environmental persistence and bioaccumulation because of their high boiling and melting points, low water solubility and electrochemical stability (Hall, Tang, Baeyens \& Dewil, 2009). Most of them are highly toxic, mutagenic and/or carcinogenic (Samanta, Singh \& Jain, 2002) and their toxicity increases with increasing molecular weight (Haritash \& Kaushik, 2009). Since they are harmful for human and living organism, their removal from the environment is very essential. Mostly particulate materials such as carbon nanomaterials, silica gels, porous nanoparticles etc., are potentially used as an adsorbents for the removal of PAHs from aqueous media (Topuz \& Uyar, 2017; Walcarius \& Mercier,2010; Yang, Zhu \& Xing, 2006), yet, electrospun polymeric nanofiber-based membranes were also studied recently by our group for the removal of PAHs from water (Celebioglu et al., 2014; Kayaci, Aytac \& Uyar, 2013). In these studies, surface modifications which are highly complicated steps were performed to enhance the efficiency of nanofibrous membranes. However, fabrication of composite CA/polybenzoxazine electrospun nanofibrous membrane via electrospinning and thermal curing is a simple approach providing the reproducibility of the same property membranes. Moreover, incorporation of polybenzoxazine can enhance not only the thermal/mechanical properties but also the adsorption performance of electrospun CA nanofibrous membrane. Because, highly crosslinked structure of polybenzoxazines and the presence of benzene rings would provide both improved thermal/mechanical features and inherently hydrophobic characteristic which can form $\pi-\pi$ and hydrophobic interactions with PAH molecules to enhance their removal from aqueous media.

In this study, we aimed to get benefit from the attractive properties of the polybenzoxazines in order to enhance especially the thermal and mechanical properties of the CA nanofibers while maintaining or even enhancing the adsorption performance by simply producing CA/polybenzoxazine composite nanofibrous membranes through electrospinning and curing process. Different compositions of the CA and BA-a blend solutions were prepared and uniform composite nanofibrous membranes were able to produced via electrospinning. However, the effective cross-linking of nanofibrous membrane through thermal curing was achieved by additional crosslinker (i.e. citric acid) in CA/ benzoxazine blend electrospinning solution, which is confirmed by the solubility tests. Morphological characterizations of the electrospun nanofibrous membranes in all steps (i.e. before and after curing, after solubility test) were performed. Also, thermal stability and mechanical performance of the pristine CA and composite CA/polybenzoxazine nanofibrous membranes were investigated. Moreover, the comparative adsorption performance of pristine $\mathrm{CA}$ and composite CA/polybenzoxazine nanofibrous membranes were investigated by removing model PAH molecule (i.e. phenanthrene) from the aqueous solutions. Our results demonstrated that cross-linked composite CA/polybenzoxazine nanofibrous membranes have shown some potentials to be used as filtration membrane for water purification and/or wastewater treatment due to their structural stability and enhanced thermal/mechanical properties.

\section{Experimental}

\subsection{Materials}

Paraformaldehyde (95\%), bisphenol-A (97\%), aniline (99\%), cellulose acetate (CA, Mw: 30000, 39.8 wt.\% acetyl) dichloromethane (DCM, $\geq 99 \%$ ), methanol ( $\geq 99.7 \%$ ), chloroform (99-99.4\%), FTIR grade potassium bromide (99\%) and phenanthrene (98\%) were purchased from Sigma-Aldrich. Deuterated chloroform $\left(\mathrm{CDCl}_{3}, 99.8 \%\right)$ was obtained from Merck. Citric acid (CTR) and sodium hydroxide $(\mathrm{NaOH}$, $98 \%$, small beads) was obtained from Sigma and Fluka, respectively. The water used was from a Millipore Milli-Q Ultrapure Water System. All the materials were used without any purification.

\subsection{Synthesis of benzoxazine monomer}

Benzoxazine monomer (BA-a) was synthesized from bisphenol-A ( $0.05 \mathrm{~mol})$, aniline $(0.1 \mathrm{~mol})$ and paraformaldehyde $(0.2 \mathrm{~mol})$ by employing solventless method (Ning and Ishida, 1994) (Fig. S1). All the reactants were mixed in $50 \mathrm{ml}$ vial and reaction mixture was stirred for $1 \mathrm{~h}$ at $110^{\circ} \mathrm{C}$ in open atmosphere. BA-a was produced as yellow highly dense viscous liquid. Then, it kept at room temperature to cool down and transformed into a solid form. The BA-a was further dissolved in chloroform and then chloroform was removed completely by using rotary evaporator system. For purification procedure, the resulting BA-a was washed with $3 \mathrm{M} \mathrm{NaOH}$ and residual reactants were removed and the final product was dried in vacuum oven over night and yellow 
powder was obtained. Molecular weight of the BA-a was measured with high resolution mass and results are $(\mathrm{M}+\mathrm{H})^{+}$calculated: 234.1489; observed: 234.1477 (Fig. S2). The chemical structure of the synthesized BA-a was confirmed by ${ }^{1} \mathrm{H}$ NMR and FTIR spectroscopy (see supporting information, Fig. S3-4).

\subsection{Electrospinning of nanofibers}

The homogenous solutions $(5 \mathrm{ml})$ of cellulose acetate (CA) and blend of CA/BA-a were prepared in DCM/methanol mixture solvent system (DCM: methanol; 4:1, v/v). Concentration of the CA solution was determined to be $12 \%$ and the solutions of the CA/BA-a blend were prepared by using the different amount of the BA-a ( 2 and $5 \% \mathrm{w} / \mathrm{v}$, with respect to solvent), CA (10 and $12 \% \mathrm{w} / \mathrm{v}$, with respect to solvent) and additionally, CTR ( $1 \% \mathrm{w} / \mathrm{v}$, with respect to solvent) was added to CA/ BA-a solutions as a cross-linker. Homogenous and clear solutions were obtained after $2 \mathrm{~h}$ stirring of CA/BA-a/CA blend. The solutions were taken in $3 \mathrm{ml}$ syringes with metallic needle of $0.4 \mathrm{~mm}$ outer diameter and the syringe was positioned horizontally on the syringe pump (KD Scientific, KDS 101). Then the positive electrode of the high voltage power supply (Spellman, SL60) was clamped to the metal needle. Metal plate collector covered by a piece of aluminum foil was placed across the horizontally positioned syringe to collect nanofibers. Electrospinning was performed by applying $15 \mathrm{kV}$ voltage, $1 \mathrm{ml} / \mathrm{h}$ flow rate and $10 \mathrm{~cm}$ distance from the collector in the completely enclosed plexiglas box and the inside temperature and relative humidity were around $24{ }^{\circ} \mathrm{C}$ and $18 \%$, respectively. Nanofibrous membranes were obtained as free-standing materials with a circular dimension having diameter of around $12 \mathrm{~cm}$ after detaching from aluminum foil. After the electrospinning, the collected nanofibers were dried over night at room temperature inside the fume hood in order to remove any residual solvent.

\subsection{Curing studies of electrospun nanofibrous membranes}

In order to obtain cross-linked membranes, the thermal curing studies of CA/BA-a and CA/BA-a/CTR nanofibrous membranes were carried out at different temperatures by step-wise curing. Initially, nanofibrous membranes were put in the oven heated at $150{ }^{\circ} \mathrm{C}$ and kept for one hour at that temperature. Then, nanofibrous membranes were subjected to step-wise heating at 175,200 and $225^{\circ} \mathrm{C}$ for $1 \mathrm{~h}$ each temperature to provide opening and cross-linking of the oxazine ring in the structure of BA-a in the nanofiber matrix.

\subsection{Adsorption performance of electrospun nanofibrous membranes for phenanthrene removal}

Adsorption performance of the nanofibrous membranes was evaluated by using phenanthrene as a model PAH molecule. The proper amount of phenanthrene was dissolved in acetonitrile and then diluted with water at the maximum solubility level of phenantherene and $1.0 \mathrm{ppm}$ phenanthrene solution was obtained. The $50 \mathrm{mg}$ nanofibrous membranes $(\sim 6 \times 6 \mathrm{~cm})$ from each sample were immersed in $30 \mathrm{ml}$ phenanthrene solutions. Then, $0.5 \mathrm{ml}$ of each solution was withdrawn to measure phenanthrene concentration in the solution and replenished with same amount of water at pre-determined time intervals. Removal efficiency (\%) and adsorption capacity of the membranes calculated by Eqs. (1) and (2), respectively (See supporting information for equations). Kinetics of the phenanthrene adsorption onto the nanofibrous membranes were studied by using pseudo-first-order and pseudo-seconorder models are represented by Eqs. (3) and (4), respectively (See supporting information for equations).

\subsection{Measurements and characterization}

High resolution mass spectrum of BA-a monomer was measured with Agilent Technologies 6224 TOF LC/MS. The chemical structure of the synthesized BA-a monomer was confirmed by proton nuclear magnetic resonance $\left({ }^{1} \mathrm{H}\right.$ NMR, Bruker Advance III $\left.400 \mathrm{MHz}\right)$ spectrometer. Samples were prepared by dissolving about $20 \mathrm{mg} / \mathrm{ml} \mathrm{BA-a}$ in $\mathrm{CDCl}_{3}$. Fourier transform infrared (FTIR, Bruker-VERTEX70) spectrometer was employed to confirm the ring-opening reactions and crosslinking of BA-a monomer during the curing process. FTIR spectra were obtained with 64 scans at a resolution of $4 \mathrm{~cm}^{-1}$ within $4000-400 \mathrm{~cm}^{-1}$ range. Samples were prepared by grinding with $\mathrm{KBr}$ in a ratio around 3:100 (3 mg sample: $100 \mathrm{mg} \mathrm{KBr}$ ) and then compressed to form discs. A rheometer (Physica MCR 301, Anton Paar) equipped with a cone/plate accessory (D:25 mm) was used to measure the viscosity of the solutions in DCM: methanol $(4: 1, \mathrm{v} / \mathrm{v})$ with a constant shear rate of $50 \mathrm{~s}^{-1}$. Results recorded with 40 rotations (per second one rotation) of the cone/plate for each sample and average of these results was considered as a viscosity value of one measurement. Viscosity measurement was repeated three times for each solutions and the average \pm standard deviation of these measurements was calculated for each solution. Scanning electron microscope (SEM, Quanta 200 FEG, FEI) was used to investigate the morphology of the nanofibers. Samples were coated with $5 \mathrm{~nm} \mathrm{Au/Pd}$ (PECS-682) prior to the SEM imaging. Average fiber diameters (AFD) of nanofibers were calculated from 50 fibers. Thermal properties of the nanofibers were investigated with thermogravimetric analyzer (TGA, Q500, TA Instruments) by starting from room temperature to $800{ }^{\circ} \mathrm{C}$ under nitrogen atmosphere at a heating rate of $20^{\circ} \mathrm{C} / \mathrm{min}$. Dynamic mechanical analyzer (DMA, Q800 TA Instruments) equipped with tensile fixture was used to measure the mechanical properties of the nanofibrous membranes. Rectangular shaped samples were prepared with dimensions of around $\sim 4 \mathrm{~mm}$ (gap) $\times \sim 3.0 \mathrm{~mm}$ (width) $\times \sim 0.02 \mathrm{~mm}$ (thickness) for nanofibrous membranes. Stress-strain curve of the three replicate from each nanofibrous membranes were obtained at $0.025 \mathrm{~N} / \mathrm{min}$ force ramp. Ultimate stress and elongation at break of electrospun nanofibers were determined from the obtained stress-strain curves and Young's modulus was calculated from the linear region of these curves. The average and standard deviation of these values were calculated. High performance liquid chromatography (HPLC, Agilent 1200 Series) was used to investigate the adsorption performance of nanofibrous membranes by removal of phenanthrene from aqueous solution. The detection of phenanthrene was performed with Zorbax Eclipse XDB-C18 column $5 \mu \mathrm{m}(150 \times 4.6 \mathrm{~mm})$ and it was detected at $254 \mathrm{~nm}$ wavelength. Acetonitrile (100\%) was used as mobile phase at a flow rate of $0.3 \mathrm{ml} /$ min and the injection volume was kept at $10 \mu \mathrm{l}$. The amount of phenanthrene remaining in the solution was determined from the area of phenanthrene peak observed in HPLC chromatograms. The peak area under curves was converted to concentration (ppm) according to the calibration curve. The calibration curve of phenanthrene was prepared by using stock solutions in 4 different concentrations; $1.8 \mu \mathrm{g} / \mathrm{ml}$, $0.9 \mu \mathrm{g} / \mathrm{ml} 0.45 \mu \mathrm{g} / \mathrm{ml}$, and $0.23 \mu \mathrm{g} / \mathrm{ml} \mathrm{R}^{2}$ was calculated as 0.99 . This experiment was repeated three times for each sample. The results were reported as the average \pm standard deviation of phenanthrene concentration remaining in the solution.

\section{Results and discussion}

\subsection{Structural characterization of benzoxazine monomer (BA-a)}

BA-a monomer was synthesized from bisphenol-A, aniline and paraformaldehyde by solventless method (Fig. S1). The structure of the synthesized BA-a was confirmed by ${ }^{1} \mathrm{H}$ NMR and FTIR spectroscopy. Fig. S3 represents the ${ }^{1} \mathrm{H}$ NMR spectrum of BA-a. The characteristic benzoxazine resonances attributable to the $\mathrm{O}-\mathrm{CH}_{2}-\mathrm{N}$ and the $\mathrm{Ph}-\mathrm{CH}_{2}-\mathrm{N}$ methylene of the oxazine ring were observed at $5.36 \mathrm{ppm}$ and $4.31 \mathrm{ppm}$, respectively. Existence of these peaks with the 1:1 ratio was the evidence for the synthesis of high purity BA-a. The characteristic peaks of the benzoxazine structure were observed in the FTIR 

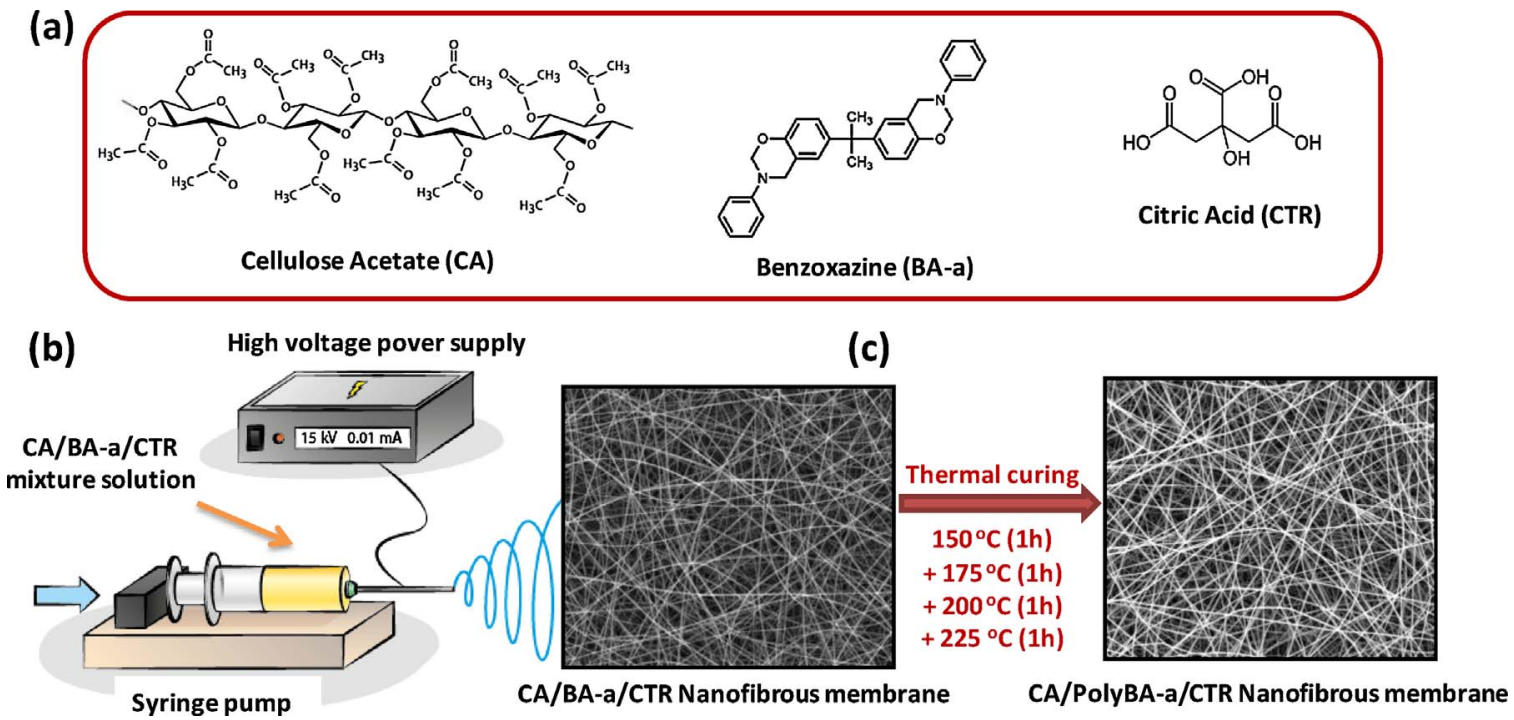

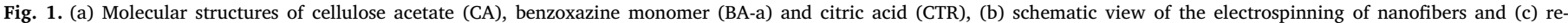
presentation of curing process of nanofibrous membrane.

spectra of BA-a shown in Fig. S4. The peaks appearing at $1496 \mathrm{~cm}^{-1}$ and $946 \mathrm{~cm}^{-1}$ ascribed to the in-plane $\mathrm{C}-\mathrm{C}$ stretching and the out-ofplane $\mathrm{C}-\mathrm{H}$ deformation of tri-substituted benzene ring, respectively. The peak appearing at $1232 \mathrm{~cm}^{-1}$ is due to asymmetric stretching mode of $\mathrm{C}-\mathrm{O}-\mathrm{C}$ in the oxazine ring. These findings correlate with the ${ }^{1} \mathrm{H}$ NMR results indicating the synthesis of BA-a was achieved.

\subsection{Electrospinning of nanofibrous membranes}

Homogenous solutions of the cellulose acetate (CA) and blend solutions of CA and BA-a (CA/BA-a) were prepared in DCM/methanol $(4: 1, \mathrm{v} / \mathrm{v})$ solvent mixture system. Molecular structures of the compounds used for the preparation of the electrospinning solutions and schematic view of the electrospinning of nanofibers are shown in Fig. 1a and b, respectively. As we reported previously, bead-free and uniform CA nanofibers can be obtained at $12 \% \mathrm{CA}(\mathrm{w} / \mathrm{v})$ concentration (Celebioglu et al., 2014), therefore, we used our previously optimized conditions for the electrospinning of CA nanofibers. Here, in order to produce uniform and bead-free composite CA/BA-a, different compositions of the CA/BA-a solutions were prepared by varying the concentration of CA (10 and $12 \% \mathrm{w} / \mathrm{v}$, with respect to solvent) and BA-a (2 and $5 \% \mathrm{w} / \mathrm{v}$, with respect to solvent) in the blend solution. These solutions are denoted as CAx:BA-ay, where $\mathrm{x}$ is the concentration of the $\mathrm{CA}$ and $\mathrm{y}$ is the concentration of the BA-a. As a result, three different compositions of the CAx:BA-ay were obtained as follow; CA10/BA-a2, CA10/BA-a5 and CA12/BA-a2. SEM images of the nanofibrous membranes obtained from these solutions revealed the successful production of bead-free and uniform nanofibrous membranes from all composition of CAx/BA-ay solutions (Fig. 2). Pristine CA nanofibrous membrane was also electrospun for comparison studies (Fig. 2a). In order to investigate the effect of the BA-a content on the fiber morphology, viscosity measurement were performed and average fiber diameter (AFD) of the nanofibers were calculated from SEM images. The viscosity and AFD of the electrospun nanofibers are summarized in Table S1. AFD of pristine CA nanofibers obtained from $12 \%(\mathrm{w} / \mathrm{v})$ CA solution was calculated as $720 \pm 295 \mathrm{~nm}$ and viscosity of this solution was measured as $0.496 \pm 0.131$ Pa.s. Slightly thinner nanofibers with the AFD of $620 \pm 195 \mathrm{~nm}$ were obtained from CA10/BA-a2, presumably because of the lower CA concentration (Fig 2c-d). Also the viscosity $(0.449 \pm 0.077)$ of this solution was lower than the pristine CA solution due to the decrease in the CA polymer concentration which resulted in lower viscosity and consequently slightly thinner nanofibers were obtained. In the second composition, CA concentration was maintained $10 \%(\mathrm{w} / \mathrm{v})$ as previous one and BA-a amount was increased as much as possible in the mixture solution in order to provide the formation of nanofibers. Here, $5 \%(\mathrm{w} / \mathrm{v})$ was determined to be the highest BA-a amount that allows the formation of uniform bead-free nanofibers, yet, no significant difference was observed on the AFD of CA10/BA-a5 nanofibers which is measured as $630 \pm 250 \mathrm{~nm}$ (Fig 2ef). In addition, viscosity of the CA10/BA-a5 solution was measured as $0.454 \pm 0.145 \mathrm{~Pa}$-s which is very similar to the CA10/BA-a2 solution. Here, It was observed that increasing the BA-a content while maintaining the CA concentration constant did not affect the viscosity much because BA-a is a small molecule and therefore, the AFD of the CA10/ BA-a2 and CA10/BA-a5 nanofibers were quite similar. On the other hand, increasing the CA concentration to $12 \%(\mathrm{w} / \mathrm{v})$ and using $2 \%(\mathrm{w} /$ v) BA-a yielded higher solution viscosity (0.501 $\pm 0.021 \mathrm{~Pa} \cdot \mathrm{s})$, hence, slightly thicker nanofibers with the AFD of $760 \pm 245 \mathrm{~nm}$ were obtained (Fig 2g-h). Although, there is not much significant difference of AFD among these samples, the results suggest that the CA concentration has more dominant affect on the fiber diameter rather than the BA-a amount. This result is anticipated since CA consist of high molecular weight polymer chains whereas BA-a monomer is a small molecule.

\subsection{Structural and morphological characterization of nanofibrous membranes}

Curing studies were performed in the temperature range which the polymerization and cross-linking of the benzoxazine monomer (BA-a) into polybenzoxazine (PolyBa-a) can be achieved without decomposing the CA polymer. The cross-linking of the CA/BA-a nanofibrous membranes was achieved by thermal curing and as a result composite CA/ PolyBA-a nanofibrous membranes were obtained. The polymerization/ cross-linking temperature of the BA-a is up to $240{ }^{\circ} \mathrm{C}$ (Ning and Ishida, 1994), hence, CA10/BA-a2, CA10/BA-a5 and CA12/BA-a2 nanofibrous membranes were thermally cured in an oven by step-wise thermal curing at the following temperatures; $150,175,200$ and $225^{\circ} \mathrm{C}$ for $1 \mathrm{~h}$ at each temperature value (Fig. 1c). During the thermal curing, BA-a monomer was polymerized/cross-linked (PolyBA-a) by ring-opening and cross-linking reactions and CA10/PolyBA-a2, CA10/PolyBA-a5 and CA12/PolyBA-a2 nanofibrous membranes were obtained. SEM images revealed that the fibrous morphology was preserved for all of the CA/ PolyBA-a samples after the curing process (Fig. 3).

However, these CA/PolyBA-a nanofibrous membranes could not retain the fiber structure and dissolved in time when they were immersed in good solvents for CA (i.e: DCM/methanol solvent mixture in 

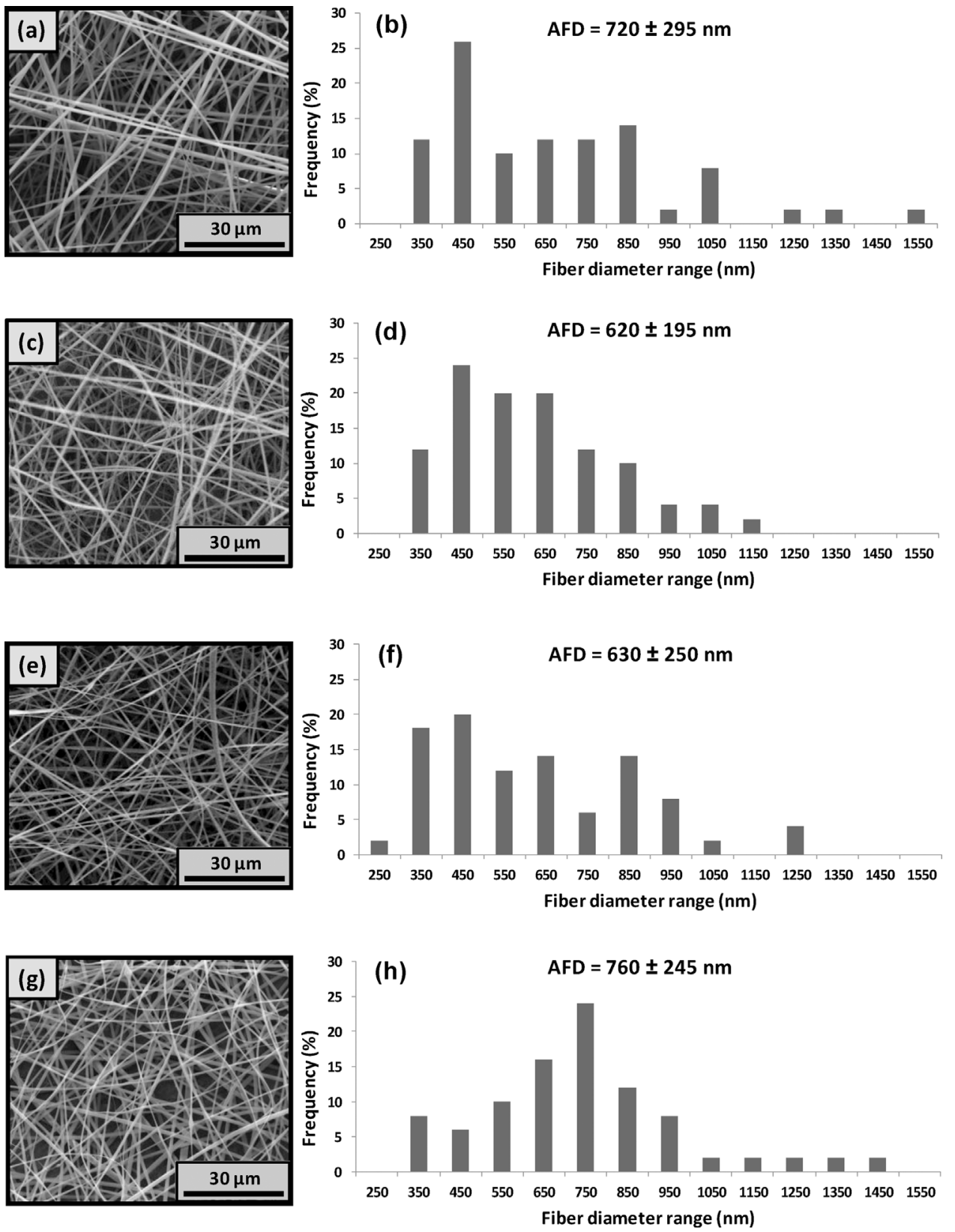

Fig. 2. The representative SEM images and fiber diameter distribution graphs of (a,b) pristine CA, (c, d) CA10/BA-a2, (e, f) CA10/BA-a5 and (g,h) CA12/ BA-a2 nanofibrous membranes, respectively. which the electrospinning was performed). This finding revealed that the total cross-linking of nanofibrous membranes was not achieved by curing of benzoxazine in the CA fiber matrix. Therefore, in order to overcome the solubility problem of these nanofibrous membranes in these organic solvents, we come up with a strategy by achieving the cross-linking of not only benzoxazine but also CA polymer matrix. Hence, citric acid (CTR) was used as a cross-linking agent. CTR is polycarboxyl organic compound that acts as a cross-linking agent because of the carboxyl groups in its molecular structure. It is reported that carboxylic acids cross-link the hydroxyl groups in cellulose and the cross-linking reaction occur temperature range $165-175^{\circ} \mathrm{C}$ (Yang, Wang \& Kang, 1997). As mentioned above, uniform CA/BA-a nanofibrous membranes were produced from all compositions tested, yet, CA10/BA-a5 composition was chosen for the production of nanofibrous membrane due to the highest amount of the BA-a. The CTR $(1 \%, w / v$, with respect to solvent) was added to the CA10/BA-a5 mixture solution and bead-free uniform nanofibers were able to electrospun from this blend system. Nanofibrous membrane obtained from this composition was denoted as CA10/BA-a5/CTR1. The thinner nanofibers were obtained from CA10/BA-a5/CTR1 having AFD of $435 \pm 165 \mathrm{~nm}$ when compared to CA10/BA-a5 nanofibers (Fig 4a-b). The CA10/BA-a5/ CTR1 nanofibrous membrane was also cured by step-wise at 150, 175, 200 and $225^{\circ} \mathrm{C}$, as a result, CA10/PolyBA-a5/CTR1 was obtained. SEM image of the CA10/BA-a5/CTR1 showed that the addition of the CTR did not affect the production of uniform nanofibrous membranes and even after the curing, nanofibrous membrane preserved its fibrous structure (Fig. 4c-d).

Furthermore, in order to investigate the cross-linking of the CA10/ PolyBA-a5/CTR1 nanofibrous membrane, solubility tests were performed. Although, CA10/PolyBA-a5 nanofibrous membrane without CTR dissolved immediately in DCM and methanol, CA10/PolyBA-a5/ CTR1 nanofibrous membrane preserved the mechanical integrity and fibrous morphology even overnight immersing which is the evidence of the successful cross-linking of the fiber matrix (Fig. S5). To further investigate the cross-linking provided by thermal curing and also molecular structural changes occurring during the thermal curing, FTIR spectroscopy technique was used. Generally, characteristic absorbance peaks of the benzoxazine ring in the FTIR spectrum were investigated 

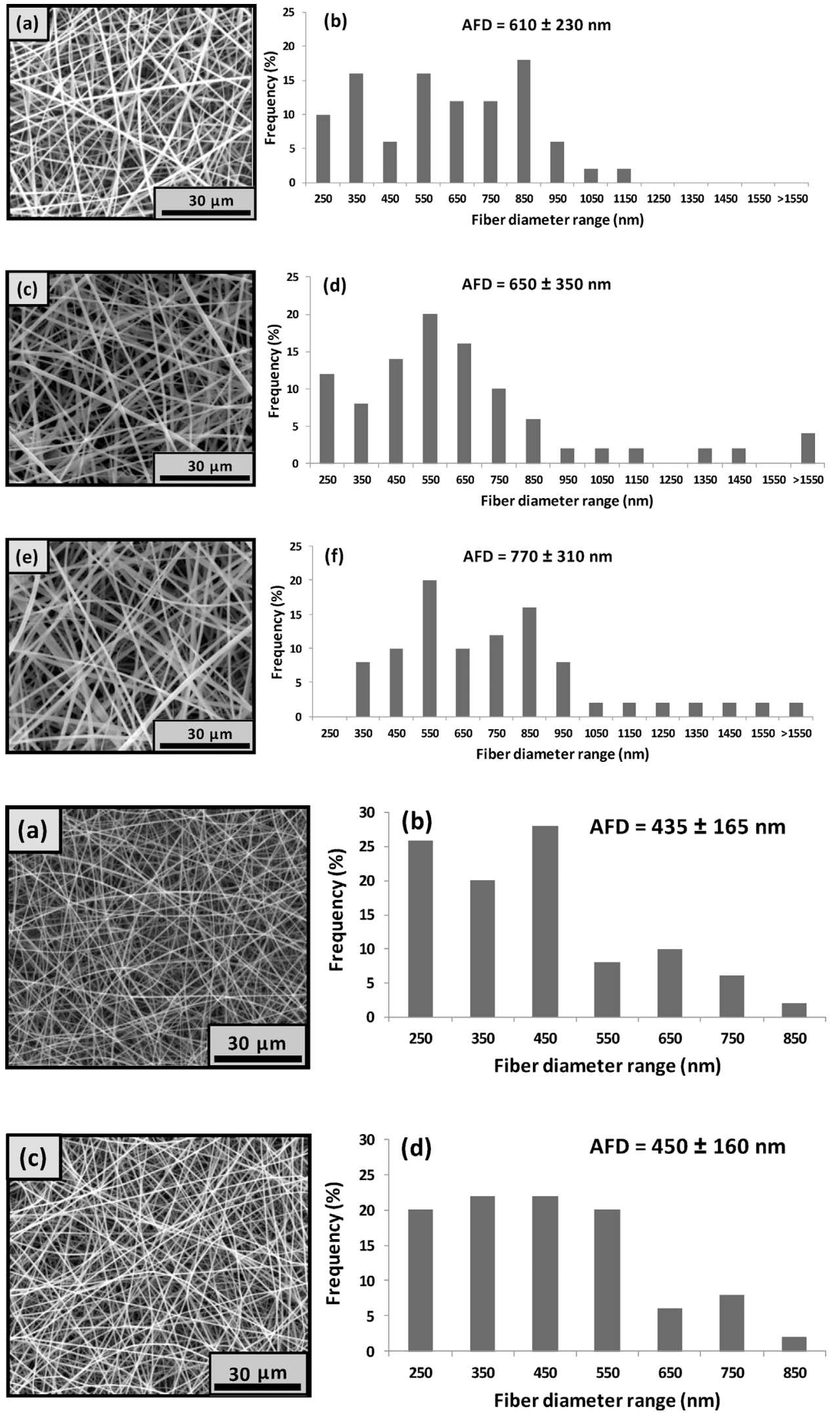

Fig. 3. The representative SEM images and fiber diameter distribution graphs of (a, b) CA10/PolyBAa2, (c, d) CA10/PolyBA-a5 and (e, f) CA12/PolyBA-

a2 nanofibrous membranes, respectively.
Fig. 4. The representative SEM images and fiber diameter distribution graphs of $(a, b)$ CA10/BA-a5/ CTR1 and (c, d) CA10/PolyBA-a5/CTR1 nanofibrous membranes, respectively. during the thermal curing studies. Characteristic benzoxazine peaks for the BA-a were observed at $946 \mathrm{~cm}^{-1}$ (out-of-plane $\mathrm{C}-\mathrm{H}$ deformation mode of the benzene ring attached to oxazine ring), $1232 \mathrm{~cm}^{-1}$ (asymmetric stretching of $\mathrm{C}-\mathrm{O}-\mathrm{C}$ in the oxazine ring) and $1496 \mathrm{~cm}^{-1}$ (in-plane $\mathrm{C}-\mathrm{C}$ streching mode of the tri-substituted benzene ring). As it is known, heat treatment provides the opening of oxazine rings and the intensity of these characteristic benzoxazine peaks decreases with the thermal curing. In order to differentiate the origin of the peaks, FTIR spectra of BA-a and pristine CA, CA10/BA-a5/CTR1, CA10/PolyBA-a5/ CTR nanofibrous membranes were recorded (Fig. 5). Firstly, 


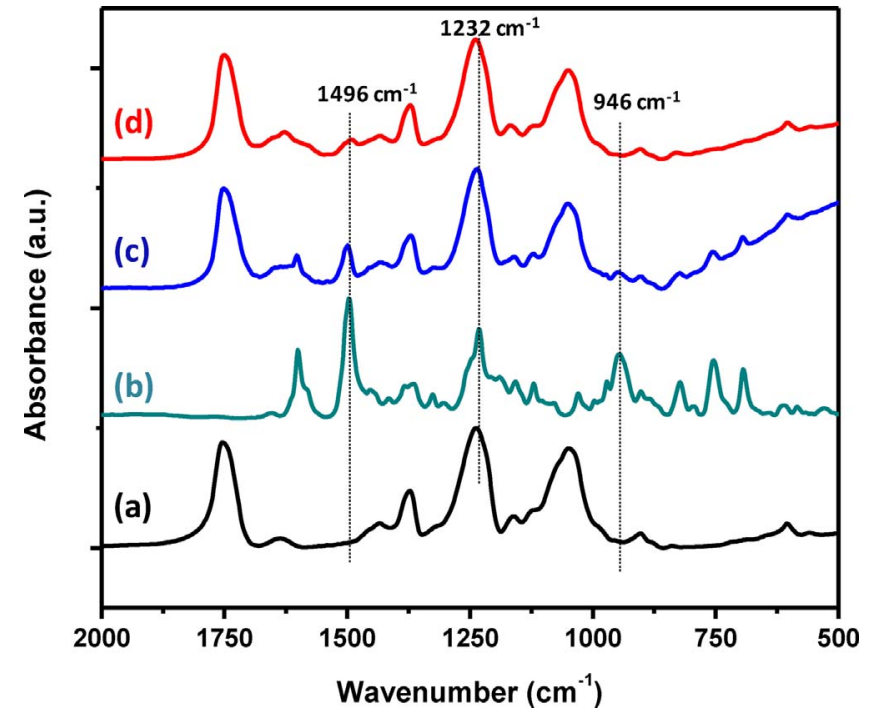

Fig. 5. FTIR spectra of (a) pristine CA nanofibrous membrane, (b) BA-a, (c) CA10/BA-a5/ CTR1 nanofibrous membrane and (d) CA10/PolyBA-a5/CTR1 nanofibrous membrane cured step-wise at $150,175,200$ and $225^{\circ} \mathrm{C}$.

characteristic benzoxazine peaks which are not overlapping with the CA peaks were determined in order to investigate the ring opening and cross-linking reactions. As it is observed, while peaks at $946 \mathrm{~cm}^{-1}$ and $1496 \mathrm{~cm}^{-1}$ belonging to the BA-a is clearly differentiated in the FTIR spectrum of CA10/BA-a5/CTR1, peak at $1232 \mathrm{~cm}^{-1}$ is overlapping with the $\mathrm{C}-\mathrm{O}-\mathrm{C}$ stretching of the $\mathrm{CA}$, hence, peaks at $946 \mathrm{~cm}^{-1}$ and $1496 \mathrm{~cm}^{-1}$ were used to investigate ring opening reactions. After the thermal curing, these peaks were disappeared in the FTIR spectrum of CA10/PolyBA-a5/CTR1 nanofibrous membrane confirming the achievement of the cross-linking and polymerization of BA-a. Although polymerization of BA-a was clearly identified by FTIR, cross-linking of CA with CTR was not differentiated due to the overlapping peaks. CA has very strong absorption peak in the range of around $1690-1880 \mathrm{~cm}^{-1}$ belongs to the $\mathrm{C}=\mathrm{O}$ stretching of the ester group (Barud et al., 2008). Although, it is expected to observe formation of new $\mathrm{C}=\mathrm{O}$ stretching band of carboxyl group of CTR around $1750-1735 \mathrm{~cm}^{-1}$ and $\mathrm{C}-\mathrm{O}$ stretching band between 1000 and $1300 \mathrm{~cm}^{-1}$ in the FTIR spectrum of CA10/BA-a5/CTR1 because of the involvement of CTR to the CA and BA-a mixture, these bands could not be differentiated due to the overlapping with the absorption peaks of CA. In addition, since the amount of the CTR $(1 \%, \mathrm{w} / \mathrm{v})$ in the composition is very less when compared with the CA $(10 \%, \mathrm{w} / \mathrm{v})$, it is not surprise to do not observe the formation of new peaks belong to CTR. Nevertheless, it can be suggested that both polymerization/crosslinking of BA-a and cross-linking of CA in the nanofibers were achieved by thermal curing after the addition of CTR according to the solubility test results since insoluble nanofibrous membranes were obtained (Fig. S5)

Moreover, physical appearance of the nanofibrous membranes also confirms the cross-linking. As it is observed in Fig. 6, the distinct color change was observed from white to brownish yellow after thermal curing owing to the cross-linking/polymerization of the BA-a. Moreover, fluffy structure of pristine CA nanofibrous membrane has transformed to flexible film like structure and the surface of the nanofibrous membrane became smother after the formation of CA10/PolyBA-a5/ CTR1. This nanofibrous membrane was obtained as free-standing material having the flexibility by transforming its original form/shape without any deformation after bending/folding. On the other hand, although pristine CA nanofibrous membrane was also obtained as freestanding material, it became fluffier and fibers stick to each other when it is folded. In brief, CA10/PolyBA-a5/CTR1 nanofibrous membrane was more handable and flexible compared to the pristine CA nanofibrous membrane which make it more suitable for the membrane applications.

\subsection{Thermal and mechanical properties of nanofibrous membranes}

TGA was used to investigate the changes occurring in thermal properties of pristine CA nanofibrous membranes after the formation of CA10/PolyBA-a5/CTR1 nanofibrous membrane. TGA curves of these two nanofibrous membranes are given in Fig. 7a. Composite nanofibrous membrane formation did not affect the degradation temperature (Td) of the pristine CA nanofibrous membrane significantly. Td onset and $\mathrm{Td}$ maximum values of the pristine $\mathrm{CA}$ nanofibrous membrane were measured as $243^{\circ} \mathrm{C}$ and $378{ }^{\circ} \mathrm{C}$, respectively. After the CA10/PolyBAa5/CTR1 composite nanofibrous membrane formation, these values were measured as $257^{\circ} \mathrm{C}$ and $378{ }^{\circ} \mathrm{C}$, respectively. On the other hand, char yield of the pristine CA nanofibrous membrane has increased remarkably and measured as $12.2 \%$ and $24.7 \%$ for pristine CA and CA10/ PolyBA-a5/CTR1 nanofibrous membranes, respectively.

Mechanical properties of the pristine CA, CA10/BA-a5/CTR and CA10/PolyBA-a5/CTR nanofibrous membranes were studied by stressstrain analysis. Since pristine CA nanofibrous membrane has fluffy structure, it was not very easy to perform stress-strain measurement for this sample. Stress-strain curve of the as-spun pristine CA nanofibrous membrane is shown in Fig. $7 \mathrm{~b}$ which is not representing the actual elongation because of the sliding and the separation of nanofibrous layers instead of breaking the nanofibrous membrane (Fig. S6). The proper stress-strain measurement could not be performed for pristine CA nanofibrous membrane due to its fluffy structure. Yet, stress-strain measurements were able to carry out for CA10/BA-a5/CTR1 and CA10/ PolyBA-a5/CTR1 nanofibrous membranes due to their compact structure. CA10/BA-a5/CTR1 nanofibrous membrane is considered as a reference in order to investigate the mechanical properties of nanofibrous membranes after cross-linking (CA10/PolyBA-a5/CTR1) provided by thermal curing. The stress-strain curves of the electrospun nanofibrous membranes (CA10/BA-a5/CTR1 and CA10/PolyBA-a5/CTR1) before and after the cross-linking are given in Fig. $7 \mathrm{~b}$. The results show that even the addition of BA-a and CTR without the curing enhanced the mechanical properties when compared to pristine CA nanofibrous membrane. However, remarkable enhancement was observed after the cross-linking achieved by thermal curing for CA10/PolyBA-a5/CTR1 sample. Tensile strength, ultimate tensile stress and Young's modulus of the CA10/BA-a5/CTR1 nanofibrous membrane were measured as $2.42 \pm 0.75 \mathrm{MPa}, 3.54 \pm 0.96 \%, 93.01 \pm 30.79 \mathrm{MPa}$, respectively. These values for CA10/PolyBA-a5/CTR1 nanofibrous membrane were measured as $8.64 \pm 0.63 \mathrm{MPa}, 8.93 \pm 0.12 \%, 213.87 \pm 30.79 \mathrm{MPa}$, respectively. This significant enhancement in mechanical performance of pristine CA nanofibrous membrane can be attributed to the stiffening effect of the BA-a with polymerization and also cross-linking of CA nanofibrous membranes.

\subsection{PAH adsorption by nanofibrous membranes}

PAHs are organic pollutants, which threat the human health seriously due to their mutagenic and carcinogenic effect. Phenanthrene is one of the well known PAH type, thus, in this study it was chosen as a model $\mathrm{PAH}$ molecule to investigate the adsorption performance of pristine CA and CA10/PolyBA-a5/CTR1 nanofibrous membranes. Fig. 8a shows the decrease of phenanthrene concentration (\%) in time when pristine CA and CA10/PolyBA-a5/CTR1 nanofibrous membranes were being kept in phenanthrene aqueous solution. It is observed that, both pristine CA and CA10/PolyBA-a5/CTR1 nanofibrous membranes adsorbed the phenanthrene effectively. Even after $10 \mathrm{~min}$ of nanofibrous membrane dipping, pristine CA and CA10/PolyBA-a5/CTR1 nanofibrous membranes reach to 66 and $78 \%$ removal efficiency, respectively. The difference between adsorbed amount of phenanthrene by pristine CA and CA10/PolyBA-a5/CTR1 nanofibrous membranes 
(a)

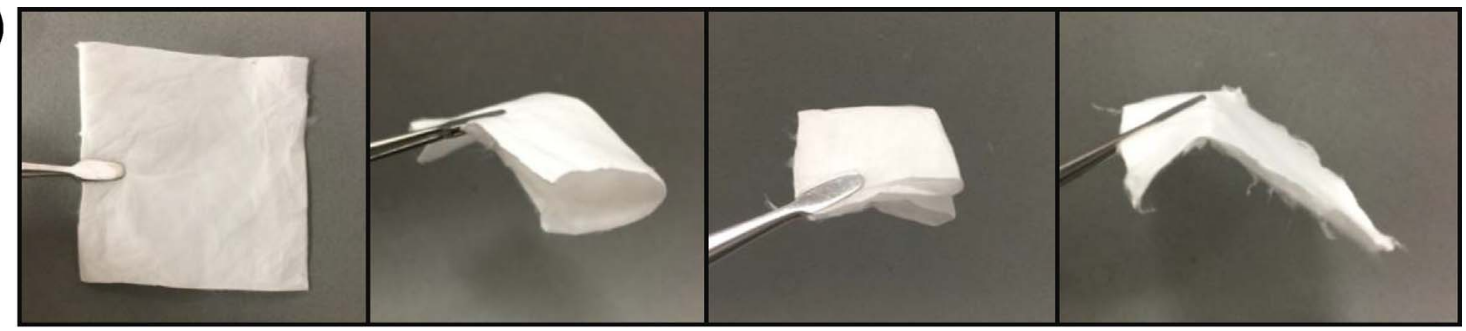

(b)

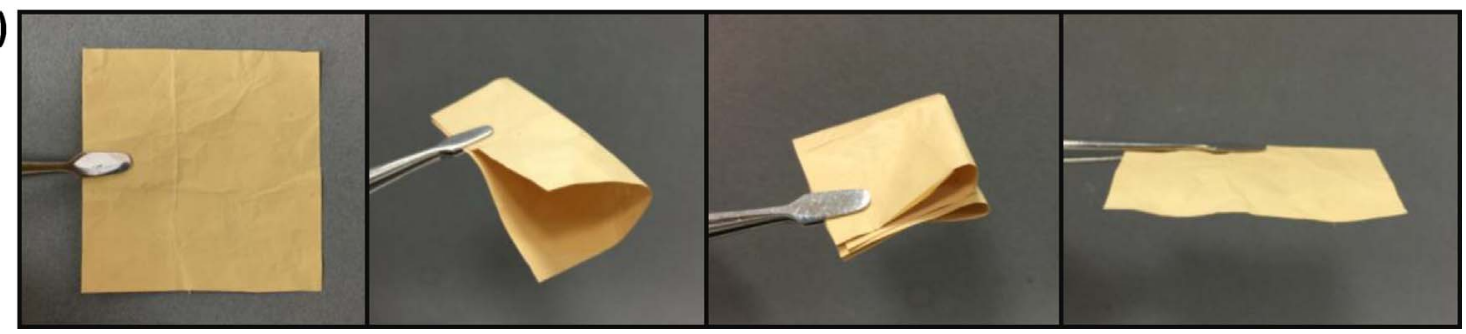

Fig. 6. Photographs of (a) pristine CA and (b) CA10/PolyBA-a5/CTR1 nanofibrous membranes.

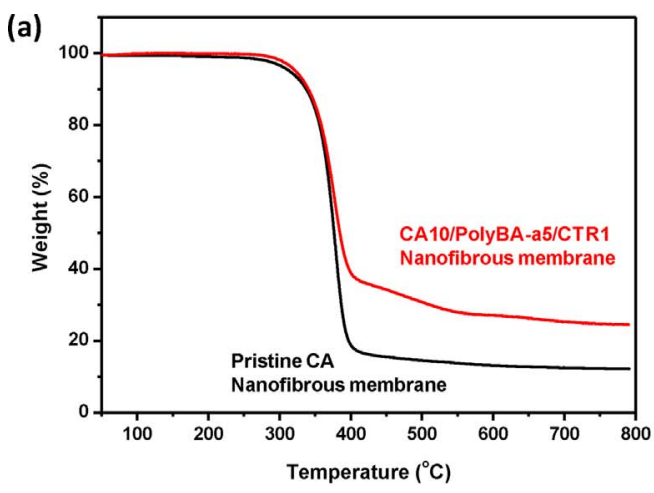

(a)

increased with time and while approaching the end of the experiment after $150 \mathrm{~min}$, removal efficiency became almost stable for both nanofibrous membranes. At the end of the experiment, removal efficiency and adsorption capacity of the CA10/PolyBA-a5/CTR1 nanofibrous membrane were calculated as $98.5 \%$ and $591 \mu \mathrm{g} / \mathrm{g}$, respectively. On the other hand, these values for the pristine CA nanofibrous membrane were determined as $92 \%$ and $552 \mu \mathrm{g} / \mathrm{g}$, respectively. As summarized in Table S2, CA10/PolyBA-a5/CTR1 nanofibrous membrane showed superior adsorption performance in a very short equilibrium time when compared with the other electrospun polymeric nanofibrous membranes (Kayaci et al., 2013; Celebioglu et al., 2014).

Adsorption is a time-dependent mechanism and investigated in terms of several processes such as chemical reaction, diffusion model and mass transfer kinetics. In this study, the kinetics of phenanthrene adsorption onto the CA and CA10/PolyBA-a5/CTR1 nanofibrous membranes were studied by using pseudo-first-order and pseudosecond-order models. Experimental data were interpreted and their validity verified by linear fitting plots. The best-fit model was determined based on the linear regression correlation coefficient $\left(\mathrm{R}^{2}\right)$ values. Fig. $8 \mathrm{~b}$ demonstrates a plot of the linearized form of the pseudofirst order model in Eq. (3) for the adsorption of phenanthrene onto nanofibrous membranes (See supporting information for Eq. (3)). $\mathrm{R}^{2}$ values for the linear plots of $\log \left(\mathrm{q}_{\mathrm{e}}-\mathrm{q}_{\mathrm{t}}\right)$ against time from the pseudofirst-order rate law are 0.9409 and 0.9086 for pristine CA and CA10/ PolyBA-a5/CTR1 nanofibrous membranes, respectively. Fig. 8c shows a plot of the linearized form of the pseudo-second order model in Eq. (4) for the adsorption of phenanthrene onto nanofibrous membranes (See supporting information for Eq. (4)). $\mathrm{R}^{2}$ values for the linear plots of $\mathrm{t} / \mathrm{qt}$ against time from the pseudo-second order rate law are 0.9995 and 0.9993 for contact times of $180 \mathrm{~min}$ for pristine CA and CA10/PolyBA-
a5/CTR1 nanofibrous membranes, respectively. This suggests that the pseudo-second order model provides the best correlation of the data indicating the chemical interactions are influential during the adsorption process.

The higher removal efficiency of CA10/PolyBA-a5/CTR1 nanofibrous membrane presumably resulted from the incorporation of the BAa to the hydrophilic CA nanofibrous membrane. Because, after thermal curing, BA-a polymerized by ring-opening and formed highly crosslinked structure with benzene rings (Fig. S7) which provided hydrophobic characteristic to the pristine CA nanofibrous membrane. Since phenanthrene is a hydrophobic molecule, most probably higher hydrophobic interactions occurred between CA10/PolyBA-a5/CTR1 nanofibrous membrane and phenanthrene molecule than pristine CA nanofibrous membrane. In addition, benzene rings found in the structure of PBA-a, consequently in the structure of CA10/PolyBA-a5/CTR1 nanofibrous membrane may provided additional interactions such as $\pi-\pi$ stacking between CA10/PolyBA-a5/CTR1 nanofibrous membrane and phenathrene. It is well known that, $\pi-\pi$ bonding interaction has been used to explain the adsorption of organic molecules with a $\mathrm{C}-\mathrm{C}$ double bond or a benzene ring (Wu et al., 2011). Also, it was observed that, both pristine CA and CA10/PolyBA-a5/CTR1 nanofibrous membranes preserved their fiber structure after the filtration experiment while the mechanical integrity of the CA10/PolyBA-a5/CTR1 nanofibrous membrane was better than the CA nanofibrous membrane. That is, CA10/ PolyBA-a5/CTR1 nanofibrous membrane preserves the original physical form perfectly after the filtration test whereas CA nanofibrous membrane was physically deformed a bit. (Fig. S8). 


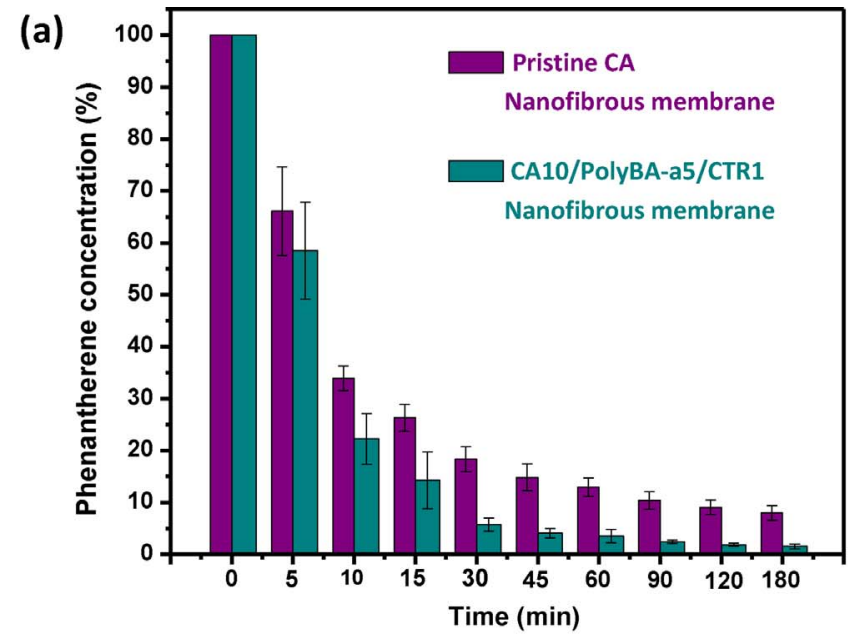

(b)

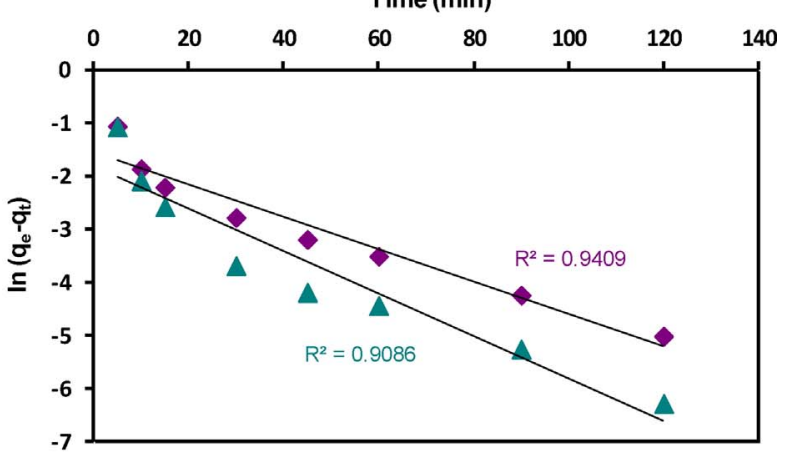

(c)

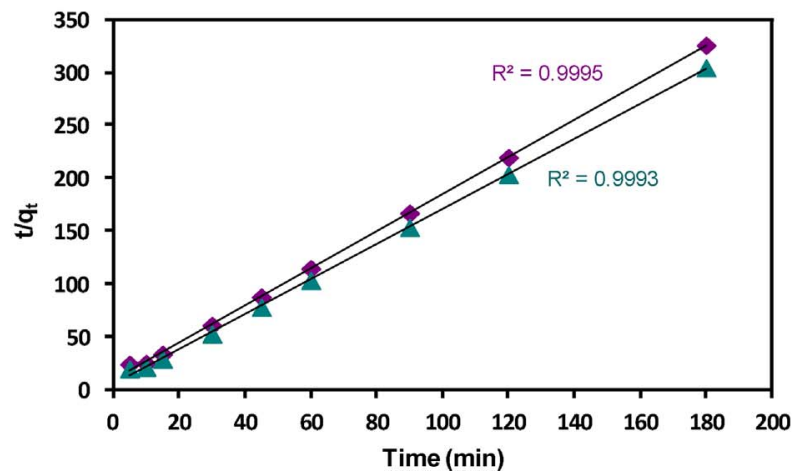

Fig. 8. The time dependent phenanthrene removal efficiency of nanofibrous membranes, (b) Pseudo-first order and (c) Pseudo-second order adsorption kinetics of phenanthrene onto $(\$)$ Pristine CA and ( $\mathbf{\Delta}$ ) CA10/PolyBA-a5/CTR1 nanofibrous membranes.

\section{Conclusion}

Here, thermal/mechanical properties and adsorption performance of the pristine CA nanofibrous membrane were enhanced by producing cross-linked composite nanofibrous membrane of CA with polybenzoxazine. Initially, we achieved to produce CA/BA-a nanofibrous membrane by varying the concentration of CA and BA-a in the electrospinning solution. After that, CTR was added as a cross-linking agent into the CA10/BA-a5 mixture solution which has the highest amount of BA-a allowing the production of nanofibers and as a result CA10/BAa5/CTR1 nanofibrous membrane was obtained. Structural characterization of the nanofibrous membranes were performed by FTIR spectroscopy revealing the opening and the cross-linking of the oxazine ring in the structure of the CA10/BA-a5/CTR1 were achieved by thermal curing and solvent resistive cross-linked composite CA10/PolyBA-a5/ CTR1 nanofibrous membrane was successfully produced. Thermal properties of the cross-linked composite CA10/PolyBA-a5/CTR1 nanofibrous membrane was improved when compared to pristine CA nanofibrous membrane. That is, thermal decomposition value of pristine CA nanofibrous membrane was increased slightly and the char yield increased significantly by producing cross-linked composite membrane. More importantly, the mechanical properties of the cross-linked CA10/ PolyBA-a5/CTR1 composite nanofibrous membrane were significantly enhanced when compared to pristine CA nanofibrous membrane. Finally, as a proof-of-concept study, molecular adsorption performance of pristine CA and cross-linked CA10/PolyBA-a5/CTR1 composite nanofibrous membrane were investigated by phenantherene removal from aqueous solution. It was observed that, cross-linked CA10/PolyBA-a5/ CTR1 composite nanofibrous membrane has shown removal of $98.5 \%$ of the initial concentration of the phenanthrene after $3 \mathrm{~h}$, whereas pristine CA nanofibrous membrane has removed $92 \%$ of that. Our results reveals that cross-linked electrospun CA/polybenzoxazine composite nanofibrous membranes can be very promising filtering materials for water purification and/or wastewater treatment due to their structural stability and enhanced thermal/mechanical properties.

\section{Acknowledgements}

Dr. T. Uyar acknowledges The Turkish Academy of Sciences Outstanding Young Scientists Award Program (TUBA-GEBIP) for partial funding. Y. Ertas acknowledges TUBITAK (2211-C) for the PhD student scholarship.

\section{Appendix A. Supplementary data}

Supplementary data associated with this article can be found, in the online version, at http://dx.doi.org/10.1016/j.carbpol.2017.08.127.

\section{References}

Abedini, R., Mousavi, S. M., \& Aminzadeh, R. (2011). A novel cellulose acetate (CA) membrane using TiO2 nanoparticles: Preparation: Characterization and permeation study. Desalination, 277, 40-45.

Ahmad, A., Waheed, S., Khan, S. M., E-Gul, S., Shafiq, M., Farooq, M., et al. (2015). Effect of silica on the properties of cellulose acetate/polyethylene glycol membranes for reverse osmosis. Desalination, 355, 1-10.

Ahmad, A., Jamshed, F., Riaz, T., Sabad, E. G., Waheed, S., Sabir, A., et al. (2016). Selfsterilized composite membranes of cellulose acetate/polyethylene glycol for water desalination. Carbohydrate Polymers, 149, 207-216.

Ahmed, F. E., Lalia, B. S., \& Hashaikeh, R. (2015). A review on electrospinning for membrane fabrication: challenges and applications. Desalination, 356, 15-30.

Arthanareeswaran, G., Devi, T. S., \& Raajenthiren, M. (2008). Effect of silica particles on cellulose acetate blend ultrafiltration membranes: Part I. Separation and Purification Technology, 64, 38-47.

Barud, H. S., De Araújo Júnior, A. M., Santos, D. B., De Assunção, R. M., Meireles, C. S. Cerqueira, D. A., et al. (2008). Thermal behavior of cellulose acetate produced from homogeneous acetylation of bacterial cellulose. Thermochimica Acta, 471, 61-69.

Bhardwaj, N., \& Kundu, S. C. (2010). Electrospinning: a fascinating fiber fabrication technique. Biotechnology Advances, 28, 325-347.

Celebioglu, A., Demirci, S., \& Uyar, T. (2014). Cyclodextrin-grafted electrospun cellulose acetate nanofibers via Click reaction for removal of phenanthrene. Applied Surface Science, 305, 581-588.

Chae, H.-R., Lee, J., Lee, C.-H., Kim, I.-C., \& Park, P.-K. (2015). Graphene oxide-embedded thin-film composite reverse osmosis membrane with high flux, anti-biofouling, and chlorine resistance. Journal of Membrane Science, 483, 128-135.

Chen, S.-L., Huang, X.-J., \& Xu, Z.-K. (2011). Functionalization of cellulose nanofiber mats with phthalocyanine for decoloration of reactive dye wastewater. Cellulose, 18, 1295-1303.

Choi, H. G., Son, M., Yoon, S., Celik, E., Kang, S., Park, H., et al. (2015). Alginate fouling reduction of functionalized carbon nanotube blended cellulose acetate membrane in forvvard osmosis. Chemosphere, 136, 204-210.

Corobea, M. C., Muhulet, O., Miculescu, F., Antoniac, I. V., Vuluga, Z., Florea, D., et al. (2016). Novel nanocomposite membranes from cellulose acetate and clay-silica nanowires. Polymers for Advanced Technologies, 27, 1586-1595.

Dasgupta, J., Chakraborty, S., Sikder, J., Kumar, R., Pal, D., Curcio, S., et al. (2014). The effects of thermally stable titanium silicon oxide nanoparticles on structure and performance of cellulose acetate ultrafiltration membranes. Separation and Purification Technology, 133, 55-68.

Ebert, K., Fritsch, D., Koll, J., \& Tjahjawiguna, C. (2004). Influence of inorganic fillers on the compaction behaviour of porous polymer based membranes. Journal of Membrane Science, 233, 71-78. 
El Badawi, N., Ramadan, A. R., Esawi, A. M. K., \& El-Morsi, M. (2014). Novel carbon nanotube-cellulose acetate nanocomposite membranes for water filtration applications. Desalination, 344, 79-85.

El-Din, L. A. N., El-Gendi, A., Ismail, N., Abed, K. A., \& Ahmed, A. I. (2015). Evaluation of cellulose acetate membrane with carbon nanotubes additives. Journal of Industrial and Engineering Chemistry, 26, 259-264.

Goh, P., Ng, B., Lau, W., \& Ismail, A. (2015). Inorganic nanomaterials in polymeric ultrafiltration membranes for water treatment. Separation \& Purification Reviews, 44, 216-249.

Hall, S., Tang, R., Baeyens, J., \& Dewil, R. (2009). Removing polycyclic aromatic hydrocarbons from water by adsorption on silicagel. Polycyclic Aromatic Compounds, 29, $160-183$.

Haritash, A., \& Kaushik, C. (2009). Biodegradation aspects of polycyclic aromatic hydrocarbons (PAHs): a review. Journal of Hazardous Materials, 169, 1-15.

Hegab, H. M., \& Zou, L. (2015). Graphene oxide-assisted membranes: fabrication and potential applications in desalination and water purification. Journal of Membrane Science, 484, 95-106.

Huang, Z. H., Li, W. J., Liu, Z. L., \& Zhang, Y. (2015). One pot blending of biopolymerTiO2 composite membranes with enhanced mechanical strength. Journal of Applied Polymer Science, 132.

Ishida, H., \& Agag, T. (2011). Handbook of benzoxazine resins. Elsevier.

Ji, F., Li, C., Tang, B., Xu, J., Lu, G., \& Liu, P. (2012). Preparation of cellulose acetate/ zeolite composite fiber and its adsorption behavior for heavy metal ions in aqueous solution. Chemical Engineering Journal, 209, 325-333.

Kayaci, F., Aytac, Z., \& Uyar, T. (2013). Surface modification of electrospun polyester nanofibers with cyclodextrin polymer for the removal of phenanthrene from aqueous solution. Journal of Hazardous Materials, 261, 286-294.

Kumari, A., Sarkhel, G., \& Choudhury, A. (2013). Effect of polyvinylpyrrolidone on separation performance of cellulose acetate-polysulfone blend membranes. Journal of Macromolecular Science Part a-Pure and Applied Chemistry, 50, 692-702.

Mahendran, R., Malaisamy, R., \& Mohan, D. (2002). Cellulose acetate and epoxy resin blend ultrafiltration membranes: preparation, characterization, and application. Journal of Macromolecular Science-Pure and Applied Chemistry A, 39, 1025-1035.

Mahendran, R., Malaisamy, R., \& Mohan, D. (2004). Preparation: characterization and effect of annealing on performance of cellulose acetate/sulfonated polysulfone and cellulose acetate/epoxy resin blend ultrafiltration membranes. European Polymer Journal, 40, 623-633.

Miller, D., Dreyer, D., Bielawski, C., Paul, D., \& Freeman, B. (2016). Surface modification of water purification membranes: A review. Angewandte Chemie International Edition, $56,4662-4711$

Ning, X., \& Ishida, H. (1994). Phenolic materials via ring-opening polymerization: synthesis and characterization of bisphenol-A based benzoxazines and their polymers. Journal of Polymer Science Part A: Polymer Chemistry, 32, 1121-1129.

Persson, K. M., Gekas, V., \& Trägårdh, G. (1995). Study of membrane compaction and its influence on ultrafiltration water permeability. Journal of Membrane Science, 100 $155-162$.

Radha, K. S., Shobana, K. H., Tarun, M., \& Mohan, D. (2014). Studies on sulfonated styrene acrylonitrile and cellulose acetate blend ultrafiltration membranes. Desalination and Water Treatment, 52, 459-469.

Razzaghi, M. H., Safekordi, A., Tavakolmoghadam, M., Rekabdar, F., \& Hemmati, M. (2014). Morphological and separation performance study of PVDF/CA blend membranes. Journal of Membrane Science, 470, 547-557.

Rodriguez, O., Peralta-Hernandez, J. M., Goonetilleke, A., \& Bandala, E. R. (2017). Treatment technologies for emerging contaminants in water: A review. Chemical
Engineering Journal, 323, 361-380.

Sabir, A., Shafiq, M., Islam, A., Sarwar, A., Dilshad, M. R., Shafeeq, A., et al. (2015). Fabrication of tethered carbon nanotubes in cellulose acetate/polyethylene glycol400 composite membranes for reverse osmosis. Carbohydrate Polymers, 132, 589-597.

Safarpour, M., Khataee, A., \& Vatanpour, V. (2015). Thin film nanocomposite reverse osmosis membrane modified by reduced graphene oxide/TiO 2 with improved desalination performance. Journal of Membrane Science, 489, 43-54.

Saliba, R., Gauthier, H., Gauthier, R., \& Petit-Ramel, M. (2000). Adsorption of copper (II) and chromium (III) ions onto amidoximated cellulose. Journal of Applied Polymer Science, 75, 1624-1631.

Samanta, S. K., Singh, O. V., \& Jain, R. K. (2002). Polycyclic aromatic hydrocarbons: environmental pollution and bioremediation. TRENDS in Biotechnology, 20, 243-248.

Shenvi, S., Ismail, A. F., \& Isloor, A. M. (2014). Enhanced permeation performance of cellulose acetate ultrafiltration membranes by incorporation of sulfonated poly $(1,4$ phenylene ether ether sulfone) and poly(styrene-co-maleic anhydride). Industrial \& Engineering Chemistry Research, 53, 13820-13827.

Suja, P., Reshmi, C., Sagitha, P., \& Sujith, A. (2017). Electrospun nanofibrous membranes for water purification. Polymer Reviews, 1-38.

Sun, Z. H., \& Chen, F. S. (2016). Hydrophilicity and antifouling property of membrane materials from cellulose acetate/polyethersulfone in DMAc. International Journal of Biological Macromolecules, 91, 143-150.

Taha, A. A., Wu, Y.-N., Wang, H., \& Li, F. (2012). Preparation and application of functionalized cellulose acetate/silica composite nanofibrous membrane via electrospinning for Cr (VI) ion removal from aqueous solution. Journal of Environmental Management, 112, 10-16.

Tian, Y., Wu, M., Liu, R., Li, Y., Wang, D., Tan, J., et al. (2011). Electrospun membrane of cellulose acetate for heavy metal ion adsorption in water treatment. Carbohydrate Polymers, 83, 743-748.

Topuz, F., \& Uyar, T. (2017). Cyclodextrin-functionalized mesostructured silica nanoparticles for removal of polycyclic aromatic hydrocarbons. Journal of Colloid and Interface Science, 497, 233-241.

Voisin, H., Bergström, L., Liu, P., \& Mathew, A. P. (2017). Nanocellulose-based materials for water purification. Nanomaterials, 7, 57-74.

Walcarius, A., \& Mercier, L. (2010). Mesoporous organosilica adsorbents: nanoengineered materials for removal of organic and inorganic pollutants. Journal of Materials Chemistry, 20, 4478-4511.

Wang, Y., Zhang, X., He, X., Zhang, W., Zhang, X., \& Lu, C. (2014). In situ synthesis of MnO 2 coated cellulose nanofibers hybrid for effective removal of methylene blue. Carbohydrate Polymers, 110, 302-308.

Wu, T., Cai, X., Tan, S., Li, H., Liu, J., \& Yang, W. (2011). Adsorption characteristics of acrylonitrile, p-toluenesulfonic acid: 1-naphthalenesulfonic acid and methyl blue on graphene in aqueous solutions. Chemical Engineering Journal, 173, 144-149.

Yang, C. Q., Wang, X., \& Kang, I.-S. (1997). Ester crosslinking of cotton fabric by polymeric carboxylic acids and citric acid. Textile Research Journal, 67, 334-342.

Yang, K., Zhu, L., \& Xing, B. (2006). Adsorption of polycyclic aromatic hydrocarbons by carbon nanomaterials. Environmental Science \& Technology, 40, 1855-1861.

Yin, J., Fan, H. J., \& Zhou, J. C. (2016). Cellulose acetate/poly(vinyl alcohol) and cellulose acetate/crosslinked poly (vinyl alcohol) blend membranes: preparation, characterization, and antifouling properties. Desalination and Water Treatment, 57, 10572-10584.

Ying, Y., Ying, W., Li, Q., Meng, D., Ren, G., Yan, R., et al. (2017). Recent advances of nanomaterial-based membrane for water purification. Applied Materials Today, 7 , $144-158$. 